\title{
Analytic Closed Orbit Analysis for RHIC Insertion*
}

\author{
S.Y. Lee' \\ Department of Physics, Indiana University, Bloomington, IN 47405 \\ S.Tepikian
}

Accel. Development Department, Brookhaven National Laboratory, Upton, NY 11973

\section{Abstruet}

Analytic closed orbit analysis is performed to evaluate the tulerance of quadrupole misalignment and dipole errors $\left(b_{0}, a_{0}\right)$ in the RHIC insertion. Sensitivity coefficients of these errors are tabulated for different $\beta^{*}$ values. Using these sensitivity tables, we found that the power supplies ripple of $10^{-4}$ can cause closed orbit motion of $0.05 \mathrm{~mm}$ at the IP in comparison with the rms beam size of $0.3 \mathrm{~mm}$. It is desirable to have the power supply ripple less than $10^{-5}$.

\section{Introduction}

The closed orbit error in the insertion region is of fundamental important to the collider physics. For RIIIC, the closed orbit at the high- $\beta$ triplets may also affect the dynamical aperture. Evaluation of the sensitivity on the quadrupole alignment errors and dipole excitation nr rotation angle error gives us a feeling of the alignment tolerance. Some correction schemes may also applied to obtain proper orbit control in the insertion region.

Fig. 1 shows the RHIC inserion layout. There are nine quadrupoles on both sides of the interaction point (IP). The closed orbit can result from (1) quadrupole misalignment, (2) dipole error, etc. 'This paper discusses an analylic method in the closed orbit analysis.

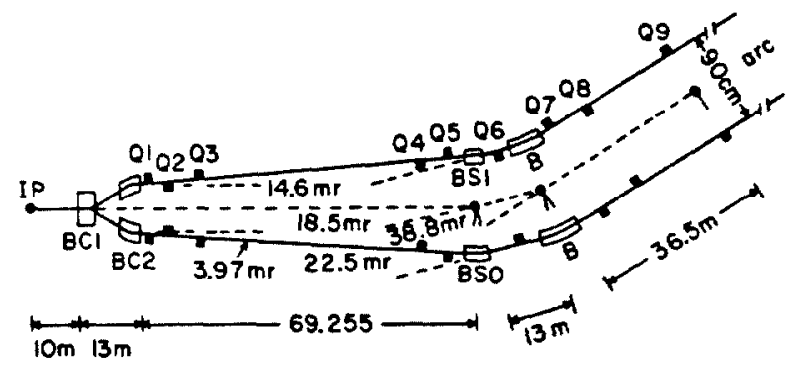

Fig. 1: Schematic Layout of a RHIC insertion.

\section{Method of Orbit Error Analysis}

For a particle in the accelerator, the equation of motion is given $b y^{\prime}$

$$
\frac{d^{2} y}{d s^{2}}+K(s) y=\frac{\Delta B(s)}{B \rho}
$$

where $y$ represents either the radial or vertical coordinates, $K(s)$ is the focusing function. For the horizontal closed orbit error, $\Delta B(s)$ arises from the vertical dipole ficld cror due to quadrupole horizontal misalignment and dipole field errors. For the vertical closed orbit error, $\triangle B(s)$ arises from the horizontal dipole field error due to quadrupole vertical misalignments and dipole rotations.

\subsection{Orbit kick due to a quadrupole}

When a single quadrupole is shifted away from the central closed orbit by $\Delta_{q}$, the angular kick due to the quadrupole is given by $-\left(y-\Delta_{q}\right) / f_{q}$ in the thin lense approximation, where $f_{q}$ is the the focal length of the quadrupole and $y$ is the actual closed orbit. For a focusing quadrupole, $f_{q}>0$ and similarly $f_{q}<0$ for a de focusing quadrupole. Thus the particle closed orbit after the quadrupole is related to the closed orbit before the quadrupole by,

$$
\left(\begin{array}{c}
y_{a} \\
y_{a}^{\prime} \\
1
\end{array}\right)=\left(\begin{array}{ccc}
1 & 0 & 0 \\
-\frac{1}{f_{a}} & 1 & \frac{\Delta_{a}}{f_{a}} \\
0 & 0 & 1
\end{array}\right)\left(\begin{array}{c}
y_{b} \\
y_{b}^{\prime} \\
1
\end{array}\right)
$$

\subsection{Closed Orbit Kick due to a Dipole}

When a particle passes through a dipole, the closed orbit is modifed by the dipole field error. Using a thin dipole approximation, we obtain then

$$
\left(\begin{array}{c}
y_{a} \\
y_{a}^{\prime} \\
1
\end{array}\right)=\left(\begin{array}{ccc}
1 & 0 & 0 \\
0 & 1 & \Delta \theta \\
0 & 0 & 1
\end{array}\right)\left(\begin{array}{c}
y_{b} \\
y_{b}^{\prime} \\
1
\end{array}\right)
$$

where $\Delta \theta$ is the dipole field error.

\subsection{The Closed Orbit Error Propagation}

The error propagation of the closed orbit is then obtained from multiplying matrices of orbil kicks discussed in previous sections and inatrices of appropriate drift spaces. The procedure is equivalent to integrating Eq.(1) along the beam line. This procedure remains valid in the thick lense calculation. 'Thin lense approximation however simplify the calculation greatly. The final closed orbit distortion can then be expressed in terms of the angular errors of dipoles and quadrupole misalignments of quadrupoles by substituting the quadrupole strengths correspondingly. The actual result of these calculations ${ }^{2}$ for $\mathrm{RHIC}$ will be discussed in the next section.

\section{Closed Orbit Kicks in a RHIC Insertion}

Table 1 lists the sensitivity coefficients for the horizontal closed orbit error at Q5, Q3, Q2, Q1 and IP as a function of the error fields discussed in section 2. As an example, table 1 gives the closed orbit deviations at $Q 3$ and IP ( for $\beta^{*}=2 \mathrm{ml}$ ) a.s,

$$
\begin{aligned}
x_{3}= & -7 x_{\theta}-123 x_{\theta}^{\prime}+2.72 \Delta_{8}-12.5 \Delta_{7}+3.5 \frac{\Delta \theta}{\theta}+ \\
& +4.26 \Delta_{\theta}+1.1 \frac{\Delta \theta_{9}}{\theta_{1}}-8.1 \tau \Delta_{5}+5.76 \Delta_{4}
\end{aligned}
$$


and

$$
\begin{aligned}
& x_{1 F}=1.3 x_{\theta}+18.1 x_{\theta}^{\prime}-0.97 \Delta_{8}+3.32 \Delta_{7}-0.85 \frac{\Delta \theta}{\theta} \\
&-0.85 \Delta_{6}-0.18 \frac{\Delta \theta_{A}}{\theta_{1}}+1.14 \Delta_{5}-0.53 \Delta_{4}-2.62 \Delta_{3} \\
& \quad+3.18 \Delta_{2}-2.37 \Delta_{1}+0.30 \frac{\Delta \theta_{c 2}}{\theta_{c 2}}+0.21 \frac{\Delta \theta_{c 1}}{\theta_{c 1}}
\end{aligned}
$$

We observe clearly that the closed orbit, $x_{3}$, at Q3 (similarly at $Q 2$ and $Q 1)$ is very sensitive to $x_{\mathbf{g}}^{\prime}$. A 0.1 mrad error in $x_{\theta}^{\prime}$ can give rise to $12 \mathrm{~mm}$ error at $Q 3$ location by assuming a perfect machine elsewhere. This sensitivity is due to betation phase advance between $Q 3$ and $Q 9$ and a large betatron function at $\mathrm{Q} 3$ position. At the same time, $x_{3}$ is also sensitive to quadrupole misuligument at $Q 7$. A $0.25 \mathrm{~mm}$ alignment in $\mathrm{Q} 7, \mathrm{Q} 6, \mathrm{Q} 5$ and $\mathrm{Q} 4$ can cause $3 \mathrm{~mm}$ rms closed orbit error at Q3. Similarly, the closed orbit at IY is sensitive to Q1-Q3 quadrupole aligmment.

Table 1: Sensitivity Coefficients of the horizontal Closed

\begin{tabular}{|c|c|c|c|c|c|c|}
\hline$\beta^{+}-5 \mathrm{~m}$ & $x_{5}$ & $x_{3}$ & $x_{2}$ & $x_{1}$ & $x_{1 P}$ & $x^{\prime}$ \\
\hline$x_{\theta}$ & -1 & -15.5 & -8.6 & -10.3 & 3.1 & 0.5 \\
\hline$x_{\theta}^{\prime}$ & -11.2 & -246 & -137 & -164 & 46.4 & 8.6 \\
\hline$\Delta_{8}$ & 1.06 & 8.57 & 4.71 & 5.62 & -1.89 & -0.3 \\
\hline$\Delta_{7}$ & -3.37 & -34.2 & -18.8 & -22.5 & 7.19 & 1.2 \\
\hline$\delta \theta$ & .79 & 8.68 & 4.8 & 5.74 & -1.8 & -0.31 \\
\hline$\Delta_{\theta}$ & .27 & 3.85 & 2.14 & 2.56 & -0.77 & -0.13 \\
\hline$\delta \theta_{\text {, }}$ & .07 & 1.63 & 0.91 & 1.1 & -0.31 & -0.06 \\
\hline$\Delta_{5}$ & & 0 & 0 & 0 & 0 & 0 \\
\hline$\Delta_{4}$ & & 5.3 & 3.03 & 3.72 & -0.65 & -0.18 \\
\hline$\Delta_{3}$ & & & -0.59 & -1.23 & -2.57 & -0.05 \\
\hline$\Delta_{2}$ & & & & 0.6 & 3.16 & 0.1 \\
\hline$\Delta_{1}$ & & & & & -2.42 & -0.1 \\
\hline$\delta \theta_{c 2}$ & & & & & 0.30 & 0.02 \\
\hline$\delta \theta_{c 1}$ & & & & & 0.21 & 0.01 \\
\hline$\beta^{\prime}=2 \mathrm{~m}$ & $x_{5}$ & $x_{3}$ & $x_{2}$ & $x_{1}$ & $x_{I F}$ & $\overline{x_{u p}^{\prime}}$ \\
\hline$\overline{x_{\theta}}$ & -0.8 & -7 & -3.8 & -4.6 & 1.3 & 0.2 \\
\hline$x_{\theta}^{\prime}$ & -2.5 & -123 & -69.1 & -83.5 & 18.1 & 4.1 \\
\hline$\Delta_{8}$ & 1.46 & 2.72 & 1.4 & 1.58 & -0.97 & -0.1 \\
\hline$\Delta_{7}$ & -3.91 & -12.5 & -6.7 & -7.8 & 3.32 & 0.45 \\
\hline$\delta \theta$ & 0.9 & 3.5 & 1.88 & 2.21 & -0.85 & -0.12 \\
\hline$\Delta_{\theta}$ & 0.64 & 4.26 & 2.34 & 2.78 & -0.85 & -0.15 \\
\hline$\delta \theta_{1}$ & 0.07 & 1.1 & 0.61 & 0.73 & -0.18 & -0.04 \\
\hline$\Delta_{5}$ & & -8.17 & -4.59 & -5.56 & 1.14 & 0.27 \\
\hline$\Delta_{4}$ & & 5.76 & 3.3 & 4.05 & -0.53 & -0.19 \\
\hline$\Delta_{3}$ & & & -0.59 & -1.23 & -2.62 & -0.06 \\
\hline$\Delta_{2}$ & & & & 0.6 & 3.18 & 0.1 \\
\hline$\Delta_{1}$ & & & & & $-2.3 \pi$ & -0.1 \\
\hline$\delta \theta_{c 2}$ & & & & & 0.30 & 0.02 \\
\hline$\Delta \theta_{c 1}$ & & & & & 0.21 & 0.01 \\
\hline
\end{tabular}
Orbit Displacement. Here $\delta \theta=\Delta \theta / \theta$ represents percentage dipole field error.

The table also gives us a guide line for the stability requirement in the power supply. As an example, we find

$$
x_{I F}=-0.85 \frac{\Delta \theta}{\theta}-0.18 \frac{\Delta \theta_{s}}{\theta_{s}}+0.30 \frac{\Delta \theta_{c 2}}{\theta_{c 2}}+0.21 \frac{\Delta \theta_{c 1}}{\theta_{c 1}}
$$

from table 1 at $\beta^{*}=2 \mathrm{~m}$. A power supply ripple will affect $\Delta \theta / \theta$ for all dipoles coherently. At $\Delta \theta / \theta \simeq 10^{-4}$, we expect the orbit will be shifted by $0.05 \mathrm{~mm}$ in comparison with the $\sigma_{\text {beam size }} \simeq 0.3 \mathrm{~mm}$ for heavy ion beam at 100 $\mathrm{GeV} / \mathrm{u}$. The effect will be very harmful to the beam life time due to the presence of beam-beam interaction. It is therefore important to achieve the power supply ripple less than $\frac{\Delta \theta}{\theta} \leq 10^{-5}$. At $\beta^{*}=0.5 \mathrm{~m}$, table 1 gives

$$
x_{i F}=-1.8 \frac{\Delta \theta}{\theta}-0.31 \frac{\Delta \theta_{x}}{\theta_{d}}+0.30 \frac{\Delta \theta_{c 2}}{\theta_{c 2}}+0.21 \frac{\Delta \theta_{c 1}}{\theta_{c 1}}
$$

At $\Delta \theta / \theta \simeq 10^{-5}$ ripple will give $\Delta x_{i F} \simeq 0.02 \mathrm{~mm}$ in comparison with the protun beam size $\sigma=0.08$ mm at 250

\begin{tabular}{|c|c|c|c|c|c|c|}
\hline$\beta^{*}=.5 \mathrm{~m}$ & $z_{5}$ & $z_{3}$ & $z_{2}$ & $z_{1}$ & $z_{1 F}$ & $z_{U E}^{\prime}$ \\
\hline$\overline{z_{\theta}}$ & -1.4 & 3.6 & 6.7 & 4.2 & -2.5 & -0.3 \\
\hline$z_{g}^{\prime}$ & 18 & -79.3 & -141. & -87.9 & 60.5 & 6 \\
\hline$\Delta_{8}$ & -4.5 & 17.08 & 30.71 & 19.11 & -12.5 & -1.28 \\
\hline$\Delta_{\tau}$ & 2.44 & -8.78 & -15.8 & -9.86 & 6.36 & 0.66 \\
\hline$\varphi$ & .62 & -1.94 & -3.53 & -2.2 & 1.35 & 0.14 \\
\hline$\Delta_{0}$ & -.27 & 0.55 & 1.03 & 0.65 & -0.31 & -0.04 \\
\hline$\varphi$. & .07 & 0.02 & 0.01 & 0 & -0.08 & 0 \\
\hline$\Delta_{5}$ & & 0 & 0 & 0 & 0 & 0 \\
\hline$\Delta_{4}$ & & -5.3 & -9.27 & -5.7 & 4.49 & 0.41 \\
\hline$\Delta_{3}$ & & & 0.59 & 0.53 & 1.4 & 0.04 \\
\hline$\Delta_{2}$ & & & & -0.6 & -6.05 & -0.22 \\
\hline$\Delta_{1}$ & & & & & 2.42 & 0.1 \\
\hline$\varphi_{c 2}$ & & & & & 0.30 & 0.02 \\
\hline$\varphi_{c 1}$ & & & & & 0.21 & 0.01 \\
\hline$\beta^{*}=2 \mathrm{~m}$ & $z_{5}$ & $z_{3}$ & $z_{2}$ & $z_{1}$ & $z_{I P}$ & $z_{1 E}^{\prime}$ \\
\hline$z_{\boldsymbol{g}}$ & -1.1 & 2.5 & 4.7 & 3 & -1.5 & -0.2 \\
\hline$z_{9}^{\prime}$ & 9.4 & -40.8 & -73.1 & -45.5 & 31.8 & 3.1 \\
\hline$\Delta_{8}$ & -3.21 & 9.31 & 17.05 & 10.72 & -6.34 & -0.69 \\
\hline$\Delta_{7}$ & 1.77 & -4.52 & -8.36 & -5.27 & 2.91 & 0.33 \\
\hline$\varphi$ & 0.51 & -0.95 & -1.8 & -1.15 & 0.5 & 0.07 \\
\hline$\Delta_{\theta}$ & -0.64 & 0.42 & 0.94 & 0.64 & 0.14 & -0.02 \\
\hline$\varphi$. & 0.07 & 0.11 & 0.15 & 0.09 & -0.17 & -0.01 \\
\hline$\Delta_{5}$ & & 2.07 & 3.54 & 2.16 & -2.02 & -0.17 \\
\hline$\Delta_{4}$ & & -5.76 & -10.0 & -6.2 & 5.04 & 0.45 \\
\hline$\Delta_{3}$ & & & 0.59 & 0.53 & 1.39 & 0.03 \\
\hline$\Delta_{2}$ & & & & -0.6 & -6.01 & -0.22 \\
\hline$\Delta_{1}$ & & & & & 2.37 & 0.1 \\
\hline$\varphi_{c 2}$ & & & & & 0.30 & 0.02 \\
\hline$\varphi_{\mathbf{c 1} 1}$ & & & & & 0.21 & 0.01 \\
\hline
\end{tabular}
$\mathrm{GeV} / \mathrm{c}$. Thus the power supply stability is especially important to the proton collision mode.

Table 2: Sensitivity Coefficients of the Vertical Closed Orbit. Here $\phi$ is the dipole rotation angle.

Table 2 lists the sensitivity table for the vertical closed orbit by integrating Eq. (1) along the insertion, where the quadrupole vertical misalignment is given by $\Delta_{1}, \ldots, \Delta_{8}$ and the dipole rotation is given by $\varphi_{,} \varphi_{s}, \varphi_{c 2}$ and $\varphi_{c 1}$ for the corresponding dipoles $\mathrm{B}, \mathrm{BSI}, \mathrm{BC} 2$ and $\mathrm{BCl}$ respectively

Similar to that of the horizontal motion, the quadrupole alignment of $Q 1-Q 3$ is important to the proper collision at 
IP. Power supply ripple is also critical for the operation at $\beta^{*} \leq 2111$

\section{Closed Orbit of the Accelerator}

We have calculated the closed orbit error propagation from the begining of the insertion to the interaction point, i.e.

$$
\left(\begin{array}{c}
y_{I P} \\
y_{I F}^{\prime} \\
1
\end{array}\right)=\left(\begin{array}{ccc}
a_{11} & a_{12} & a_{13} \\
a_{21} & a_{22} & a_{23} \\
0 & 0 & 1
\end{array}\right)\left(\begin{array}{c}
y_{9} \\
y_{9}^{\prime} \\
1
\end{array}\right)
$$

where the matrix elements $a_{i j}$ may be obtained directly form Tables 1 and 2. For example, $a_{11}=3.1, a_{12}=46.4$ and $a_{13}=-1.89 \Delta_{8}+7.19 \Delta_{7}-1.8 \delta \theta+\cdots$ etc. for the horizontal closed orbit propagation at $\beta^{*}=0.5 \mathrm{~m}$. 'To obtain a proper closed orbit of the entire circular accelerntor, we shall assume that the orbit is propagated through the rest of the machine, i.e.

$$
\left(\begin{array}{c}
y_{9} \\
y_{9}^{\prime} \\
1
\end{array}\right)=\left(\begin{array}{ccc}
M_{11} & M_{12} & M_{13} \\
M_{21} & M_{22} & M_{23} \\
0 & 0 & 1
\end{array}\right)\left(\begin{array}{c}
\tilde{y}_{1 F} \\
\tilde{y}_{I F}^{\prime} \\
1
\end{array}\right)
$$

where $2 \times 2$ matrix corresponds to the propagation of betatron motion from the IP through the rest of the accelerator to the end of $Q^{9}$. The matrix elements $M_{13}, M_{23}$ are related the orbit kicks associnted with the rest of the accelerator. The total closed orbit is then obtained by multiplying matrices of Eqs. (4) and (5) with the following constraints,

$$
\tilde{y}_{\mathrm{IF}}=y_{I P} ; \quad \tilde{y}_{I P}^{\prime}=y_{I P}^{\prime} .
$$

The $2 \times 2$ matrix of the final product corresponds to the ane turn betation transfer map. They are given by

$$
\begin{gathered}
a_{11} M_{11}+a_{12} M_{21}=\cos 2 \pi \nu+\alpha^{*} \sin 2 \pi \nu, \\
a_{11} M_{12}+a_{12} M_{22}=\beta^{*} \sin 2 \pi \nu, \\
a_{21} M_{11}+a_{22} M_{21}=-\gamma^{*} \sin 2 \pi \nu, \\
a_{21} M_{12}+a_{22} M_{22}=\cos 2 \pi \nu-\alpha^{*} \sin 2 \pi \nu,
\end{gathered}
$$

where $\nu$ is the betatron tune for either horizontal or vertical betatron motion, $\alpha^{*}, \beta^{*}$ and $\gamma^{*}$ are the Courant-Snyder pararuetrization of the betatron functions at $1 P$. Normally $\alpha^{*}=0$ and $\gamma^{*}=1 / \beta^{*}$. Solving the closed orbit condition of $\mathrm{Eq} .(6)$, we obtain then

$$
\begin{aligned}
y_{I F}= & \frac{1}{2 \sin \pi \nu}\left\{\left(a_{11} M_{13}+a_{12} M_{23}+a_{13}\right) \sin \pi \nu\right. \\
& \left.+\beta^{*}\left(a_{21} M_{13}+a_{22} M_{23}+a_{23}\right) \cos \pi \nu\right\} . \\
y_{1 F}^{\prime}= & \frac{1}{2 \sin \pi \nu}\left\{-\frac{1}{\beta^{*}}\left(a_{11} M_{13}+a_{12} M_{23}+a_{13}\right) \cos \pi \nu\right. \\
& \left.+\left(a_{21} M_{13}+a_{22} M_{23}+a_{23}\right) \sin \pi \nu^{\prime}\right\} .
\end{aligned}
$$

Note here that the orbit error is enhanced by the nearness of the betatron tune to an integer. 'l'he sensitivity factor is however still proportional to the tables 1 and 2 through $a_{13}$ and $a_{23}$ coefficients in Eqs. (7) and (8).

\section{Conclusion and Discussion}

The closed orbit analysis for the RHIC insertion is analyzed in an analytic model in terms of the quadrupole alignment errors and the dipole errors. We calculate the sensitivity coefficients for various $\beta^{*}$ values. The closed orbit error becomes large at the high- $\beta$ quadrupoles, $Q 1$. Q3. To minimize these errors, one should properly align the quadrupoles in the insertion. One should also measure $x_{9}, x_{9}^{\prime}, z_{9}$ and $z_{9}^{\prime}$ so that a proper orbit correction scheme for the insertion can be established due to the fact that the closed orbit is sensitively dependent on the parameters $x_{\theta}^{\prime}$ and $z_{\theta}^{\prime}$ (see Tables 1 and 2 ).

Using the table, we can also set the folerance on the power supply ripple. At low $\beta^{*}$ value, the power supply ripple of $10^{-5}$ is critical to obtain a proper beam-beam collision.

Finally the ground motion due to the high tide, local traffic, etc. will shift quadrupole alignment. A shift of $10 \mu$ in Q1-Q3 can also cause beam movement at the IP by 0.01 mIn, which will affect the performance. Fortunately, the ground motions which does not change the relative motion of the accelerator component do not affect the luminosity The random noise is however normally small, $\simeq 1 \mu$. Thus the effect should not be important. Realistic measurement of the effect in RHIC tunnel should be confirmed.

The method described in this paper is useful in evaluating the effect of few important magnetic elements on the closed orbit distortion. Another possible application is analyzing the orbit distortion of a beam tranfer line.

- Work performed under the auspices of the U.S. Department of Energy.

† On leave of absence from Brookhaven National Labora tory

\section{Reference}

1. E.D. Courant and 11.S. Snyder, Ain. of Phys. 3, 1 (1958)

2. S.Y. Lee and S. Tepikian, AD/RHIC-64, Feb. 1990. 